\title{
Observation on the Curative Effect of Madopar Combined with Pramipexole in the Treatment of Parkinson's Diseases
}

\author{
YU Yong-ting1, YANG Min², CHEN Rui-hui ${ }^{3}$, CHEN Hong-bing ${ }^{\text {* }}$ \\ 1. Weifang Medical University, Weifang, Shandong, 261053, China \\ 2. The First People's Hospital of Jining, Jining, Shandong, 272011, China \\ 3. Rongjun Hospital, Shandong, Qingzhou, 262500, China \\ 4. Yidu Central Hospital of Weifang, Weifang, Shandong, 262500, China
}

Abstract: Parkinson's disease is a common neurological degenerative disease in the elderly. The clinical manifestations are static tremor, increased muscle tone, slow movement, abnormal posture gait, and severe symptoms of loss of self-care. Parkinson's disease has become a burden to not only patient, but also to his family and to the society. Normal human brain neurotransmitter dopamine and acetylcholine are in a balanced state, the two antagonism can maintain normal body function. The pathological changes of patients with Parkinson's disease is degeneration and apoptosis of dopaminergic neurons in striatum, resulting in a significant reduction in dopamine levels, and thus lead to an imbalance in the effects of dopamine and acetylcholine, and a decrease in dopamine resistance to acetylcholine Parkinson's disease symptoms. As such, this article will focus on the analysis of the Madopar and pramipexole in the treatment of Parkinson's disease symptoms.

Key words: madopar; pramipexole; Parkinson's disease; motor symptoms

\section{Introduction}

Parkinson's disease is common in the elderly, it not only causes serious damage to the physical and mental health of patients, but also greatly reduces the quality of life of patients. Currently, drug therapy is still the main way to treat Parkinson's disease clinically. Madopar is a typical drug for the treatment of Parkinson's disease. Short term of treatment is ideal, but long term treatment causes patient to be less responsive to the medication. Pramipexole is a dopamine receptor agonist which can effectively stimulate the brain dopamine, it prevents and reduces the disease

Copyright (C) 2017 YU Yong-ting, et.al.

doi: http://dx.doi.org/10.18686/aem.v6i1.80

This is an open-access article distributed under the terms of the Creative Commons Attribution Unported License

(http://creativecommons.org/licenses/by-nc/4.0/), which permits unrestricted use, distribution, and reproduction in any medium, provided the original work is properly cited. 
complications significantly. The complications of Parkinson's disease are due to levodopa preparations in the course of clinical treatment of black dopamine deficiency, reducing the quality of life of patients, resulting in patients with serious complications. Clinical medicine suggests that the progressive loss of dopaminergic neurons in the brain, the manner of exogenous levodopa intermittent supply, and the non-physiological activation of pulsed leads to the expression level and function of the dopamine receptor in the postsynaptic membrane Abnormalities are the main cause of complications of Parkinson's disease. In the clinical treatment, pramipexole is a new type of brain dopamine receptor agonist. Pramipexole can directly act on the brain black dopamine receptor, effectively avoid the striatum postsynaptic brain black dopamine receptor pulse generated to reduce the complications of Parkinson's disease. In addition, it effectively alleviate the clinical symptoms and it has important significance to the effective prognosis of patients.

\section{Data and Methods}

\subsection{General Information}

A total of 86 patients with Parkinson's disease who were treated between July 2015 and June 2016 were randomly assigned into two groups. In the control group (42 cases), 24 males and 18 females were aged from 58 to 71 years. Aaverage age was $(64.8 \pm 2.2)$ years; the course of disease was 2 to 5 years and the average duration was $(3.5 \pm 1.2)$ years; primary Parkinson's disease in 16 cases, Parkinson's super position syndrome in 14 cases, secondary Parkinson's syndrome in 12 cases. In the observation group (44 cases), 27 males and 17 females were aged from 61 to 75 years. Average age was $(65.2 \pm 2.6)$ years; the course of disease was 1 to 6 years, the average duration was $(3.2 \pm 1.3)$ years; primary Parkinson's disease in 17 cases, Parkinson's syndrome in 16 cases, 11 cases of secondary Parkinson's syndrome. Two groups of patients were diagnosed by clinical features and auxiliary blood, cerebrospinal fluid, SPECT, PET-CT and other aspects of the diagnosis are of early and mid-term diseases, and exclude the treatment of drug taboos and other ban bogey treatments. Patients and their families signed treatment consent. There was no significant difference between the two groups in the general data $(P>0.05)$.

\subsection{Method}

The observation group was treated with madopar and pramipexole. Madopar $62.5 \mathrm{mg}$, p.o., b.i.d. During the treatment period, the dose was adjusted to $250 \mathrm{mg} /$ time, p.o., t.i.d. Pramipexil $0.0625 \mathrm{mg}$, p.o.,b.i.d. During the treatment, the dose was adjusted to $0.5 \mathrm{mg} /$ time, p.o., t.i.d. The control group was treated with madopar alone. Madopar is used in the same amount as the observation group.

\subsection{Observation Indicators and Efficacy Evaluation Criteria}

1) Drug effects: The UPDRS III evaluation was used. The lower the score indicates the better the drug effect; 2) The improvement of depression symptoms: The HAMD evaluation was used. The lower the score indicates the lesser the severe depression and mild depression $\leq 17$ points; 3) Adverse reactions: Observation of patients with sleepiness, insomnia, nausea, vomiting, switching, psychiatric symptoms, dizziness and other adverse reactions; 4) Clinical efficacy evaluation criteria: Remarkably UPDRS III score and HAMD score were significantly reduced, and the clinical symptoms of patients improved significantly, the disease was under control; effectiveness for the UPDRS III score and HAMD score have a lesser decrease, patients still accompanied by mild clinical symptoms, and the progress of the disease slowed down; it is invalid as unable to meet the above criteria and exacerbations; Total effectiveness $=$ Remarkable + Effective.

1.4 Statistical Methods 
SPSS20.0 statistical software was used for statistical analysis. The measurement of data are expressed as average \pm standard deviation $\left(\mathrm{x}^{- \pm} \mathrm{s}\right)$, using $t$-test; count data in terms of rate (\%), using $\chi^{2}$ test; and ranking data use rank information and test. $P<0.05$ indicates that the difference was statistically significant.

\section{Results}

\subsection{Treatment effect comparison}

In the observation group, 19 cases were remarkably effective, 17 cases were effective and 8 cases were ineffective, and the total effective rate was $81.82 \%$. In the control group, 11 cases were remarkably effective, 18 cases were effective and 13 cases were ineffective, the total effective rate was $69.05 \%$. The difference between the two groups was statistically significant $(P<0.05)$. See Table 1 .

Table 1: The two groups of patients with the treatment effect comparison, $\mathrm{n}(\%)$

\begin{tabular}{|c|c|c|c|c|c|c|c|c|}
\hline Group & No. of Case & & $\begin{array}{l}\text { Remarkably } \\
\text { Effective }\end{array}$ & & iffective & & Ineffective & Total Effective \\
\hline $\begin{array}{l}\text { Observation } \\
\text { Group }\end{array}$ & 44 & 19 & (43.18) & 17 & $(38.64)$ & 8 & (18.18) & $36(81.82)$ \\
\hline $\begin{array}{l}\text { Control } \\
\text { Group }\end{array}$ & 42 & 11 & $(26.19)$ & 18 & $(42.86)$ & 13 & $(30.95)$ & $19(69.05)$ \\
\hline$\chi^{2}$ rate & & & - & & - & & - & 12.473 \\
\hline$P$ & & & - & & - & & - & $<0.05$ \\
\hline
\end{tabular}

\subsection{UPDRS scores comparison before and after treatment}

The scores of UPDRS were significantly higher in the two groups than those before treatment, the difference was statistically significant $(P<0.05)$. The difference between the scores of UPDRS after treatment and 6 months follow-up was statistically significant $(P<0.05)$. See Table 2 .

Table 2: UPDRS scores comparison between two groups of patients before and after treatment $\left(\bar{x}_{ \pm \mathrm{s}}\right)$

\begin{tabular}{|c|c|c|c|c|}
\hline Group & Stage & $\begin{array}{l}\text { Mental and emotional } \\
\text { behavior }\end{array}$ & Daily activities & Movement function \\
\hline \multirow{3}{*}{$\begin{array}{l}\text { Observation } \\
\text { Group } \\
(n=44)\end{array}$} & Before treatment & $12.2 \pm 2.2$ & $45.3 \pm 3.8$ & $48.8 \pm 4.4$ \\
\hline & After treatment & $7.2 \pm 1.8^{4}$ & $26.2 \pm 2.7^{ \pm 4}$ & $28.5 \pm 2.9^{\text {ut }}$ \\
\hline & 6 months follow-up & $6.2 \pm 1.4^{2 x}$ & $24.3 \pm 2.1^{\text {ale }}$ & $25.3 \pm 2.1^{\text {tex }}$ \\
\hline \multirow{3}{*}{$\begin{array}{l}\text { Control } \\
\text { Group } \\
(n=42)\end{array}$} & Before treatment & $12.3 \pm 2.1$ & $45.2 \pm 4.6$ & $48.6 \pm 4.9$ \\
\hline & After treatment & $8.5 \pm 2.3^{b}$ & $29.3 \pm 2.8^{b}$ & $32.5 \pm 3.3^{\mathrm{L}}$ \\
\hline & 6 months follow-up & $7.6 \pm 1.6^{\mathrm{kx}}$ & $26.6 \pm 2.2^{b x}$ & $28.5 \pm 2.4^{k x}$ \\
\hline
\end{tabular}

Note: Compared with the control group, ${ }^{\text {a }} P<0.05$; compared with this group before treatment, ${ }^{\text {b }} P<0.05$; compared with this group after treatment, ${ }^{\mathrm{c}} P<0.05$.

\section{Discussions}

Parkinson's disease is a common neuro-degenerative disease, generally only second to Alzheimer's disease. The risk of 
life-long disease is about $1 / 100$. The pathogenesis of Parkinson's disease is mainly due to the loss of substantia nigra dopamine neurons, and thus caused cortical basal ganglialoop dysfunction, leading to the occurrence of movement disorders. Currently, the etiology of Parkinson's disease is still not clear, but the study found that the occurrence of the disease and striatal dopamine content was significantly reduced, while the striatum dopamine content is usually reduced by dopaminergic neurons in the substantia nigra caused by aging, genetic factors, environmental degradation and other factors that can cause dopaminergic neuronal degeneration and death. With the growth of age, the number of dopaminergic neurons in the human brain will gradually decrease, so the elderly have a higher risk of Parkinson's disease. Due to the slow onset of Parkinson's disease, in the early stage patients mainly showed quiescent tremor, activity clumsy, muscle rigidity, slow movement and other clinical symptoms. However, in recent years, it was found that the occurrence of Parkinson's disease also lead to depression, constipation, insomnia and other issues which seriously affected the quality of life of patients. Patients with depression, will be caught in long term low morale, there will be loss of appetite, sleep disorders and other issues. Some patients will have obvious anxiety and exercise agitation which can be a hindrance to the normal life of the patient. As such, it is vital for patients with Parkinson's disease to take effective treatment accordingly. In general clinical practice, the use of drug treatment with levodopa preparations, and with the help of surgery can improve the effectiveness of drug treatment. Patients in the course of taking drug, together with habilitation and psychological treatment, can improve the clinical symptoms of patients. There is no way to cure this disease, but active drug therapy can reduce the clinical symptoms of patients and improve the quality of life of patients.

The main symptoms of Parkinson's disease are resting tremor, muscle rigidity, slow movement and postural reflexes, and often accompanied with psychiatric symptoms, autonomic neurosis, pain and other non-motor symptoms. The quality of life of patients with Parkinson's disease is seriously affected and can bring serious economic and psychological burdens to patients and their families. Parkinson's disease etiology and pathogenesis were not clear, so the current clinical treatments are of dopamine-based. However, long term use of dopamine in patients will give rise to psychiatric symptoms, morning stiffness and so on. Recent studies have found that pramipexole will also help to ease the symptoms of Parkinson's disease, and can improve the patient's depression, while reducing the amount of dopamine usage. Most of the patients with Parkinson's disease of the substantia nigra-striatum dopaminergic nerve pathway damage which leads to reduced dopamine secretion. Therefore, the main clinical treatment is to supplement dopamine, so as to slow down the course of development and ease the symptoms of Parkinson's disease. Recent studies have found that pramipexole also has the ability to ease the symptoms of Parkinson's disease, and can improve the patient's state of depression, while reducing the usage of dopamine. In general, the treatment of Parkinson's disease is still the mainly symptomatic treatment, and cannot stop disease progression. Modern clinical studies suggest that levodopa can replace the reduction of dopamine neurotransmitters in the brain to achieve the purpose of improving the clinical symptoms of Parkinson's disease, but long-term application prone to diminished efficacy, end effects, and switch phenomenon. Sole application of Madopar is limited. Pramipexole as a dopamine (DA) receptor agonist was gradually being recognized clinically. Pramipexole can reduce the usage of levodopa dose and reduce the occurrence of dyskinesia during the course of treatment of Parkinson's disease. The principle of improving Parkinson's disease is mainly achieved by increasing the amount of dopamine in the brain. Madopar is a common drug for the treatment of Parkinson's disease. Madopar is effective in the early treatment of Parkinson's disease, but with the progress of patient's condition, the dosage of drugs also increased. Patients with dyskinesia, mental disorders, stiffness, less moving, and other adverse reactions also increased. The mechanism of adverse reactions may be related to the pulsatile stimulation of the dopamine receptor. It is reported that about $60 \%$ of Parkinson's disease patients treated with levodopa after 4 to 6 years will experience exercise complications. Pramipexole is a new non-ergotamine dopamine receptor agonist that has a high 
electivity to dopamine receptors, it improves the symptoms of Parkinson's disease by stimulating D2 receptors. Pramipexole does not need to pass through the metabolic transformation of denatured DA neurons by directly stimulating the DA receptors in the striatum. No potential toxic metabolites, and will not compete with other substances to absorb and transfer into the brain. Hence, the adverse drug reactions is lighter than levodopa.

Levodopa is being the best choice for treatment of Parkinson's disease. It can achieve a certain effect within a short time, but with more side effects. Most patients prone to have adverse reactions after using the drugs. Patients with long-term usage of high dose madopar will lead to the body of the substantia nigra DA neurons degenerate and necrosis symptoms cannot be controlled, and thus trigger the end phenomenons, such as morning stiffness, dysmotility and other adverse reactions which will deteriorate the patient's condition. Pramipexole is a type of dopamine receptor agonist that can effectively delay the development of Parkinson's disease. The drug does not exert a stimulating effect on DA receptors directly with the aid of endogenous DA, thus delay the occurrence of Parkinson's complications in a certain extend. In relevant scholars study pointed out that the effect of madopar combined with pramipexole treatment of Parkinson's disease is more significant than the single madopar treatment, and is also safer.

\section{References:}

1. Wang X, Liu G, Xiao Q, Chen S. Dopamine receptor agonist pramipexole treatment of Parkinson's disease non-motor symptoms of observation [J]. Journal of Clinical Neurology, 2010,04:299-301.

2. Hua Z. Pramipexole treatment of Parkinson's disease non-exercise symptoms of observation [J] Practical Elderly Medicine, 2010,06:498-500.

3. Zheng J, Xu J, Liang Q, Ruan Z, Pan J. Dopamine receptor agonist pramipexole treatment of Parkinson's disease non-motor symptoms of observation [J]. South China National Defense Medical Journal, 2014,06:586-587.

4. Chen S. Acupuncture combined with the United States and Duoba treatment of Parkinson's disease clinical research [D]. Chinese Academy of Traditional Chinese Medicine, 2013.

5. Zhou T. Pluticaine hydrochloride treatment of Parkinson's disease dose-effect relationship analysis [D]. Dalian Medical University, 2013.

6. Zhang K, Yuan Y, Song C, Zhang L. Pramipexole hydrochloride to improve the curative effect of non-motor symptoms of Parkinson's disease [J]. Chinese Journal of Practical Neurology, 2012,14:11-14.

7. Liu Z, Pu Z, Liu Y, Yin P, Lu C, Zang Z. Medaoba combined with pramipine hydrochloride in the treatment of Parkinson's disease on the role of motor function [J]. Chinese Journal of Practical Neurology, 2015, 15:94-95. 